\title{
Designing Wearable Electronic Spectacle for Enhancing Social Impressions Emitting Sound and Smell
}

\author{
Yongsoon Choi \\ Dept. of Art \& Technology, Sogang University, Korea \\ yongsoon@sogang.ac.kr
}

\begin{abstract}
Sound and smells aid in the impression of one another. These two senses can generate stronger emotional feelings more than words. The author built a wearable electronic system that allows users to emit preset sounds and fragrances during a face-to-face encounter. The system is designed as a spectacle form factor allows a user to select her own characteristic sounds and smell identifications (IDs). The IDs are then transferred to another system when two people meet and indirectly stimulate each person with their partners sound and smell preference. The paper presents the design of the system and experimental results with human subjects examining how the system augments in building a positive impression using subtle sound and smell stimulations.
\end{abstract}

Keywords: Sound \& smell, electronic wearable glasses, fashion communication, personal branding, interpersonal interaction

\section{Introduction}

In today's hyper connected era, building meaningful personal relationships is becoming increasingly complicated. People may find it difficult to remember details of a past meeting with someone, such as name, common interests, emotion, and mood [1]. It can affect the quality of people's social relationship. We have never been as widely connected by communication, but we also see an increase in feelings of isolation and loneliness [2]. In this author's opinion, only interaction through Internet and screens is not enough for human connectivity.

The system is designed as a wearable electronic accessory to positively enhance the wearer's social relationships and connectivity by providing subtle olfactory and audio stimuli during face-to-face social encounters. Because a single smell or sound can influence people's moods, judgments and even affect their social relationships [3].

These two senses can aid in the impression of one another. It sometimes generates stronger emotional feelings than words and even awakens long forgotten memories.

This is similar in concept to the function of pheromones, which are chemicals produced by some animals that enable them to communicate and cause specific social reactions with other members of their own species. The spectacle system also helps people express their unique identity and personality to others using sound and smell from a wearable accessory during a face-to-face encounter.

The system was designed in a spectacle in order to unobtrusively stimulate a user's nose and ears. Two small sound and perfume actuators were attached on the spectacle, which were located behind the wearer's ears to aid indirect stimulation. They contained eight types of solid state perfume. Once a selected piece is heated with a wire, the perfume begins to vaporize, and the small speaker below the perfume heater helps pulsate the aroma from the sound and perfume actuator modules.

The spectacle emits sound and smell signals unique to the person the wearer meets. The sound and perfume spectacle communicates with the users smartphone via Bluetooth and the spectacle communicate with each other using Infrared Data Association (IrDA) 
transceivers located in the bridge of the spectacle. They exchange information only when the users look at each other directly in a face-to-face conversation. Once the IrDA transceiver detects the partner's spectacle, data containing their name, contact number and unique sound and perfume signature identity (ID) are exchanged between the systems.

This paper shows the design and technology of the spectacle system and the experimental results of a user study performed on 30 participants that were used to evaluate how the system can affect the user's impression during face-to-face communication using the indirect auditory and olfactory stimuli.

\section{Related Researches}

\subsection{Sound and Smell}

The brain forges a link between the sound, smell, and a memory associating the individual and the interaction. Moreover, when you encounter the smell again, the link is already there ready to elicit a memory or a mood. To understand this phenomenon, many researchers have shown that if we hear or smell something while undergoing an emotional experience, the sound or smell is woven together with the emotive memory in the same part of the brain [4-7].

According to Nayak's study [8], the sound of music affects portions of the brain, and music can be used to manage emotions and hence social interactions of individuals. Magee and Davidson [9] similarly state it affects improvement in quality of life, involvement with the environment, awareness and responsiveness, positive associations, and socialization. It improves building a positive effect on social and behavioral outcomes and encouraging social interactions [10].

Comparatively, using music to affect social relationships is well documented. However, using artificially generated smells for eliciting an emotional response is not a common practice yet [5]. In this section, the author more focus on smell researches that are related to emotion, memory, and olfactory wearable researches.

Smells can evoke strong emotional reactions. In surveys on reactions to odors, responses show that many of our olfactory likes and dislikes are based purely on emotional associations. According to Fox [11], the positive emotional effects of pleasant fragrances also affect our perceptions of other people. Unpleasant smells can also affect our perceptions and evaluations. In one study, the presence of an unpleasant odor led subjects not only to give lower ratings to photographed individuals, but also to judge paintings as less professional.

Smell can also help memory triggering functionality. Cann and Ross [12] describe a study in which $80 \%$ of men and $90 \%$ of women reported multiple odor-revived memories that show a strong link between smell and memory. Yeshurun [13] also found that the first pairing between an object and a smell had a distinct signature in the brain. The feeling of the initial smell memory in the brain was equivalent whether it was good or bad. Sacco and Sacchetti [14] trained rats to associate either a tonal sound, a flashing light, or a whiff of vinegar with electric shocks. They showed that the creatures' memories of fear and pain of being shocked were stored in parts of the brain that held information taken in by the eyes, ears or nose.

\subsection{Smell Actuator}

There are some research projects that use smell actuators for olfactory interactions. Olfoto presents a study into the use of smell for searching digital photo collections [15]. Olfoto also shows that some smells were consistently identified and useful, but some were not. The idea presented in SpotScents uses a method to project the smell to the user that reduces the breeze effect [16]. This is a more natural olfactory experience. Subsmell was a system designed to be used in conjunction with a movie [6]. The system would provide 
an olfactory stimulus of the scene depicted in the film. Emsenhuber's article describes several commercial systems that are capable of scent output used for marketing such as iSmell and Osmooze [5].

Additionally, there are some previous approaches to wearable olfactory actuators. Tillotson [17] has done extensive work looking at clothing that dynamically emits scent as an integral part of its design. She explores their use for health, wellness, and emotional applications. Narumi [18] developed Metacookie that is a system designed to be used in augmented taste through visual and smell stimuli, and Yamada [7] developed an olfactory display that sent scented air to the nose through a tube. This allowed users to freely move in a space. Several other olfactory displays are also introduced in the article by Washburn and Jones [19].

The novel idea of sound and perfume spectacle is to use the rich sense of sound and smell during face-to-face interpersonal communications to help enhance the user's social relationship. The indirect sound and fragrance stimulation from a pair of glasses can affect the user's impression of others and also create the users unique identity for others to build the recollection of memories on a user's impression during face-to-face interpersonal communication.

\section{System Design}

\subsection{Operational Description}

When a user starts a face-to-face conversation with another person, the sound ID and perfume ID are shared between each spectacle through an IrDA module located in the bridge of the spectacle. The partners ID information is used to trigger the sound and perfume actuators in the user's spectacle.

These actuators are attachable modules on the spectacle, which are located be-hind the wearer's ears for indirect stimulation. The aroma is created by heating solid perfume and emitted by the pulsating sound from the speaker that is simultaneously being actuated. This process occurs every time the pair meets. This indirect stimulation facilitates the user to express his or her unique ID during face-to-face interpersonal interactions, and awaken memories of the other person when they meet again after some time.

Additionally, as an example application for the sound and perfume spectacle system for evoking our memories and memorable interactions, a photo application was developed. It is paired with a mobile phones camera to save not only the images, but also the sound and smell information of the person in the photo. When the photo is viewed in the future, the sound and smell recorded with the image is triggered.

\subsection{Sound and Perfume Spectacle}

The sound and perfume spectacle is designed around the microcontroller board and also consists of a battery, the Bluetooth and IrDA modules, a heating and sound circuit, and two sound and perfume actuator modules. It consists of one speaker for sound and 4 channel perfume heaters in each module as shown in Figure 1. 


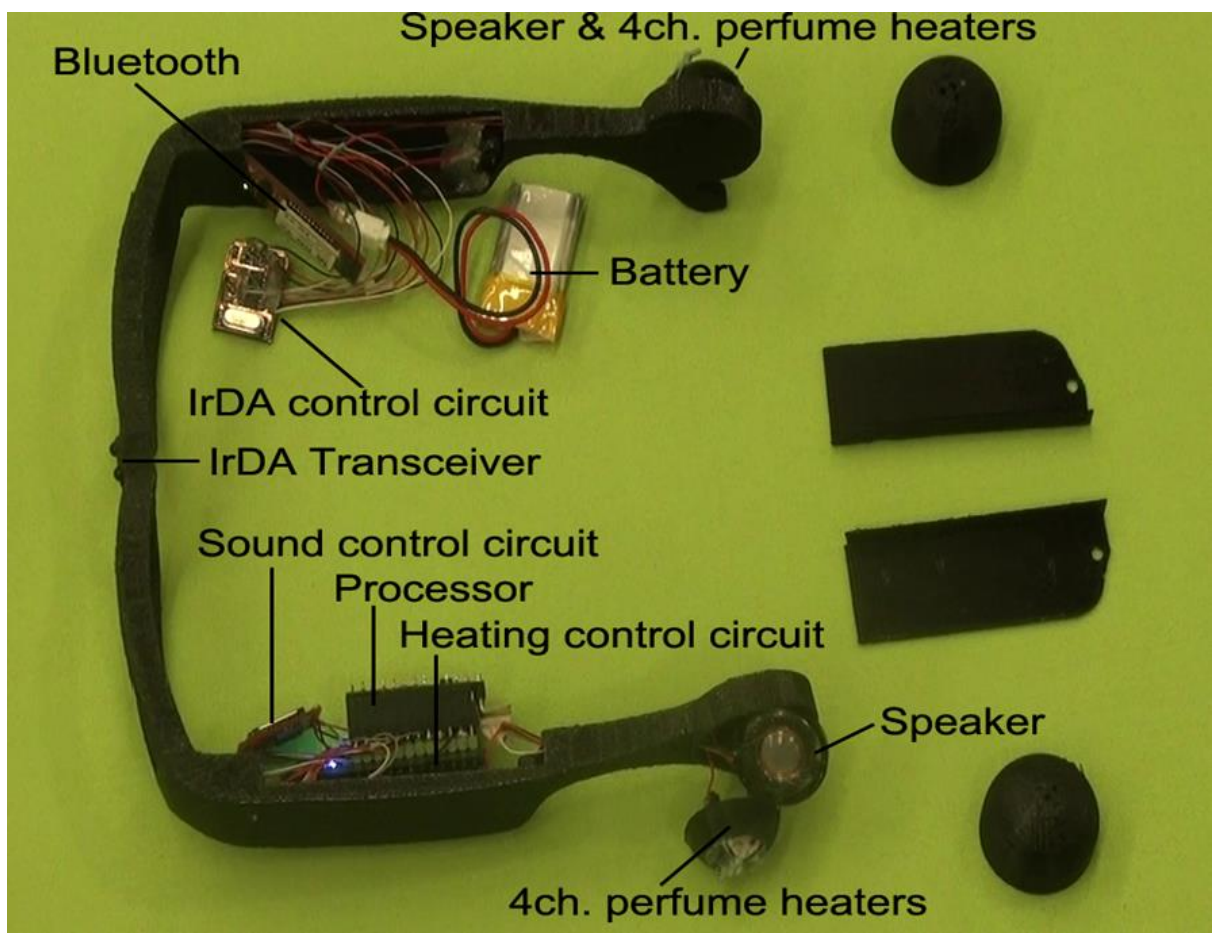

Figure 1. Components Used in the Sound Perfume Spectacle

The author uses a single board microcontroller processor $(3.3 \mathrm{~V} / 8 \mathrm{MHz})$ as the main processing unit. This processor uses eight digital pins for the perfume heaters, two digital pins for the IrDA control circuit, and four analog pins for the speakers.

The Bluetooth module provides the wireless link between a user's mobile phone and the sound and perfume spectacle. And a detachable and rechargeable Polymer lithiumion battery $(3.7 \mathrm{~V} / 1000 \mathrm{mAH})$ is also used.

The current consumption averages $40 \mathrm{~mA}$, but it can reach approximately $300 \mathrm{~mA}$ for a few seconds when heaters are used. The IrDA transceiver is used for interpersonal communication. In order to convert the electrical pulses from the IR transceiver to a microcontroller acceptable format, the author uses an infrared encoder/decoder in the spectacle which outputs the data as the Universal Asynchronous Receiver/Transmitter (UART) controller [20].

The sound circuit consists of a controller and can play a specific sound clip from a micro SD card. The clip is played using two $8 \mathrm{ohm} / 0.25 \mathrm{~W}$ speakers that are located behind the wearer's ears. For smelling, the solid essences that are easily carried and controlled in an electronic circuit are used. The four solid essences (solid fragrance, lip balm, aroma gel and solid perfume) were have been tested to choose suitable essences that have a low melting point and strong scent in a low current consuming for the sound and perfume actuator. 


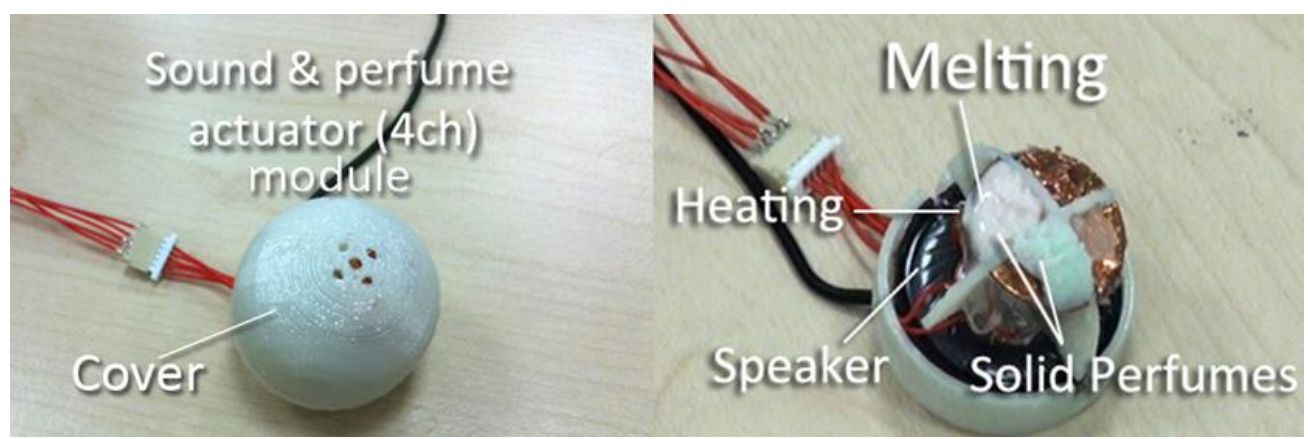

Figure 2. Sound and Perfume Actuator Module

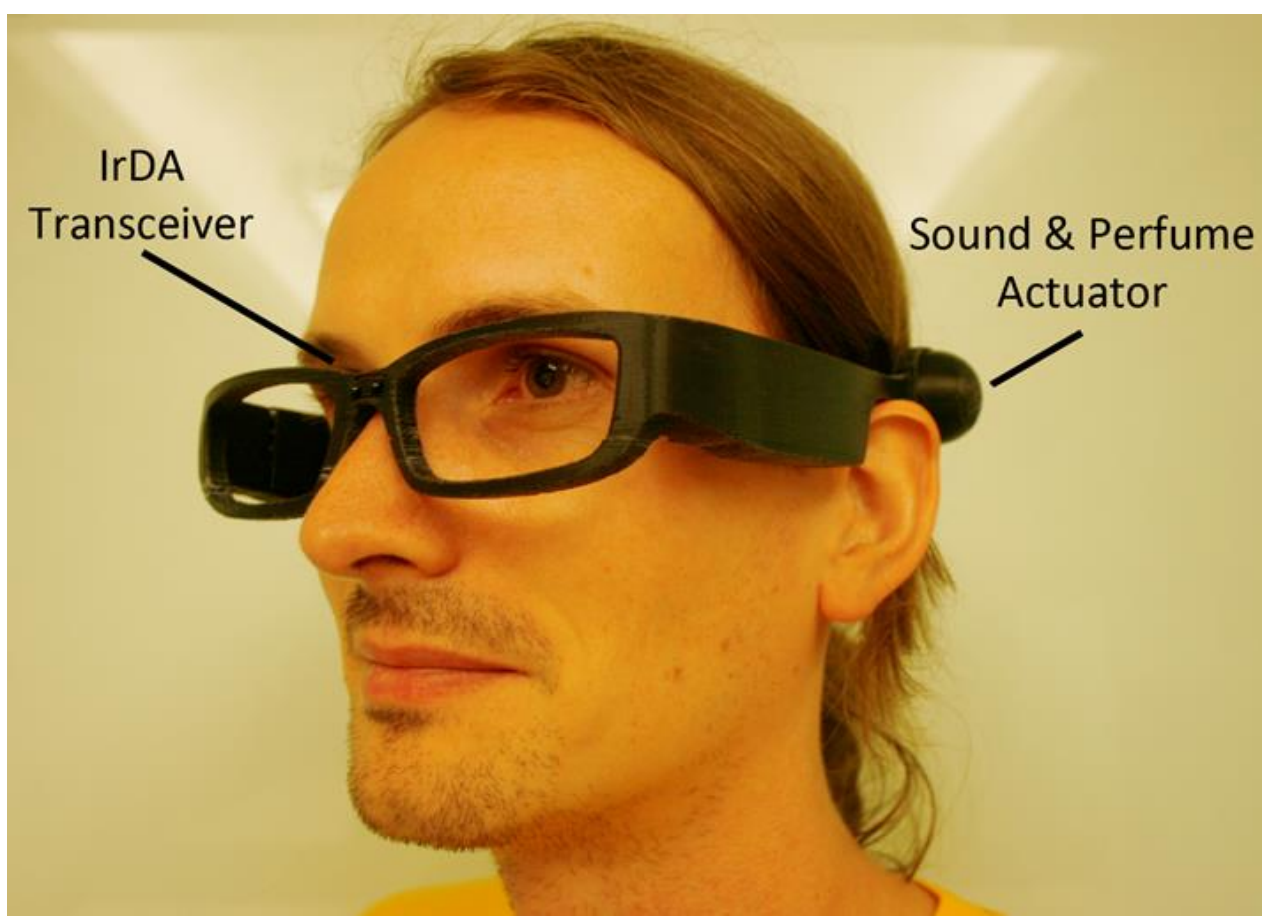

Figure 3. The Actuators are Located behind the Ears and the Irda Transceiver is Located in the Bridge of the Spectacle

According to the results in the lab, the solid perfume samples, which start to melt around $46{ }^{\circ} \mathrm{C}\left(115^{\circ} \mathrm{F}\right)$ with comparatively strong fragrance, was selected. The perfume emitter has been designed to heat solid perfume using the heat from a resistor and the construction of this actuator is shown in Figure 2.

Based on tests in the lab, a 12ohm 1/8W resistor was chosen for heating based on the average current consumption. It requires $315 \mathrm{~mA}$ and reaches the melting point for the essence in about 3.5 seconds. The aroma is gently emitted with the pulsating sound from the speaker.

Two sound and perfume actuator modules were placed behind the wearer's ears in a spectacle in order to stimulate the user's nose and ears as shown in Figure 3.

The IrDA transceiver is located in the bridge of the spectacle in order to detect and communicate with another spectacle when two people are communicating while maintaining eye-contact. 


\subsection{Mobile Phone Application}

The application was developed using the smart phone platform. The designed app helps the user to perform three functions: 1) setting the users ID, 2) contact management, and 3) photo viewer application.

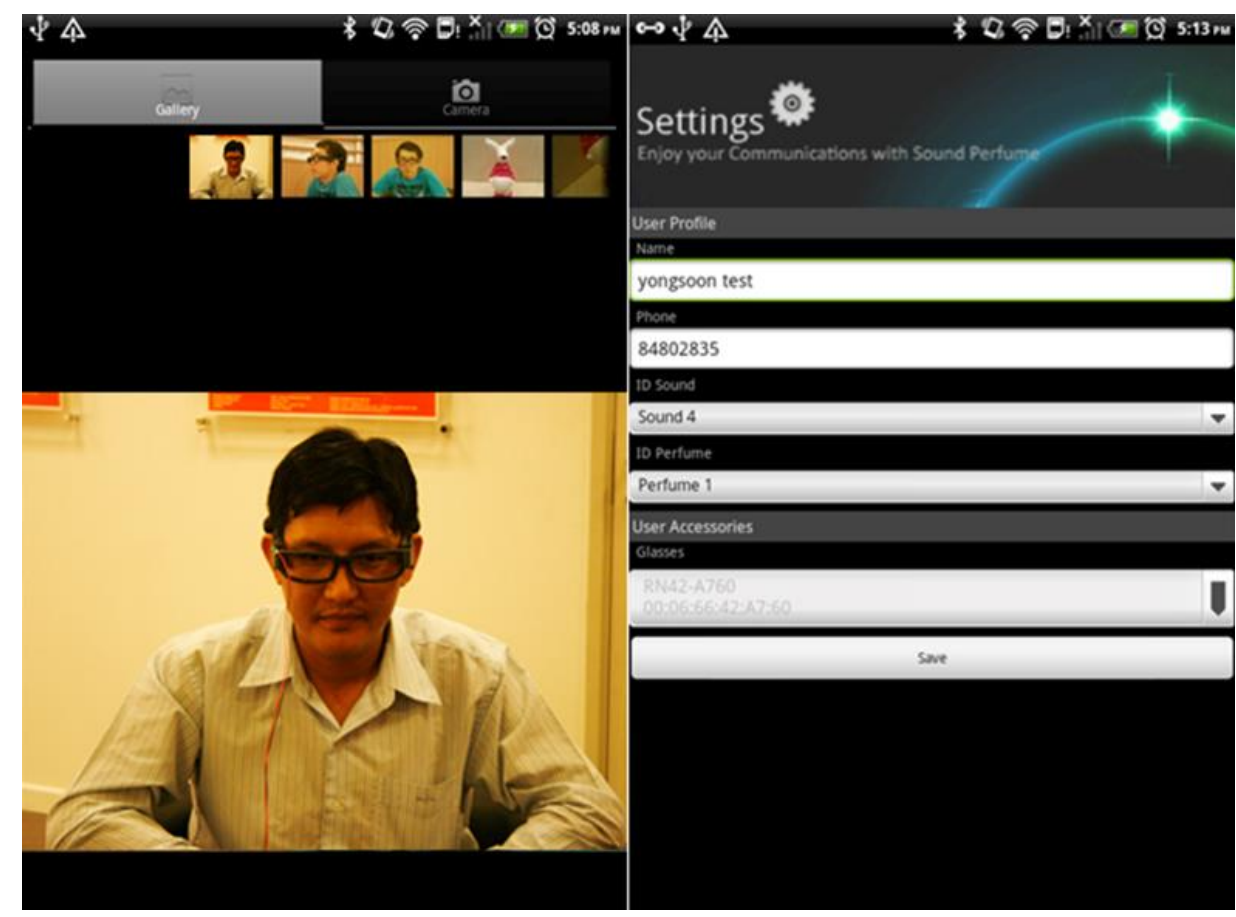
Figure 4. The Screen Shots of Mobile Application (Left - Photo Viewer Menu,
Right- Settings Menu)

1) The user can setup his sound and perfume ID in the settings menu. The IDs are associated with other details such as the name, phone number, and Bluetooth MAC address of the spectacle system in the setting menu. Once the user updates this, the preferences are sent to the spectacle where it is stored on the onboard EEPROM of the system.

2) The call log and contacts list are located in Contact menu. Once a user connects with another person via IrDA, the spectacle forward the partners ID (this includes the name, contact number, sound and perfume ID) to the mobile phone through the Bluetooth connection. The user is able to sort this data chronologically or alphabetically.

3) The photo viewer is an example application for the sound and perfume spectacle system to integrate with a photo viewer application in the mobile phone. When a user takes a picture with the other person using a mobile phone, the picture is saved with the other persons sound and perfume ID and profile information together. When the photo is viewed later, the spectacle system generates the other person's signature sound and scent automatically. The Figure 4 shows examples of screenshots of the mobile application.

\section{User Study}

The user studies were executed using the sound and perfume spectacle system to understand how the impressive auditory and olfactory stimulation from the system can help affect the wearer's impression in face-to-face communication. 
Impression is a process in which people attempt to influence the perceptions of other people [21]. The impression is formed by the subjective and emotional sensitivity connections. The assessment of the users impressions using different auditory and olfactory experiences and feelings from the sound and perfume spectacle system is a difficult task, as the users' experiences and feelings are subjective, context-dependent, and dynamic [3].

As the first step of user evaluation, the pilot user study was conducted to understand the affection of impression from the system in normal face-to-face conversation for the feedback of the main user study as the first stage. Then the main user study was executed based on the feedback of pilot study.

Ten different sound and eight different types of perfume fragrance samples were used for the tests. For sound samples, ten of Bernard Bouchard's sound samples were used from Vieilard's study [22]. It is about validating Bouchard's 56 musical excerpts that conveyed the four intended emotions (happiness, sadness, threat and peacefulness).

For smell samples, as Parry [23] states that "there have been numerous attempts at the classification of odors, all of which have been empirical and useless and most of which have been childish and absurd." It was difficult to select smell samples. The author used Zarzo and Stanton's sensory map with odor descriptors which are commonly used in the perfumery industry. In this map, proximity indicates that odors are similar, while distance reflects dissimilarity [24]. Based on this map, the author selected eight fragrance samples (floral, green, fruity, Fougre, citrus, oriental, gourmand and aquatic perfumes) for the user studies.

\subsection{First stage- pilot user study}

A pre-experiment was conducted with twenty two participants to extract sensible vocabularies of the impressions on the ten different sound and eight different perfume samples before the pilot user study.

For the pilot user study, twelve participants (six females and six males; average age twenty four years) were grouped as six couples in two groups (Group A had previous relationship with each other and Group B had no previous relationship with each other). All participants could each set their sound and smell ID using the app and then have a conversation for three minutes without any restrictions.

After the conversation with the partner, all participants were asked to freely describe their impressions on their partner. The author compared the sensible vocabularies of impressions about their partner from the users selected sound and perfume ID.

The results on pilot user study show eight participants were affected by the sound and perfume spectacle system even though three of them said they didn't recognize the sound and perfumes stimulation during the experiment.

\subsection{Feedback from the Pilot User Study}

During the pilot study, it was found that there were many other factors affecting people's impressions of their partners. For example, individual sensibility, outward appearance, fashion style, communication skill, body gestures, previous relationship, and feedback such as facial expressions and reactions during conversations, keenly effect the impression of the partner. To know the impression affection using auditory and olfactory stimuli from the spectacle system, it was decided to reduce the noise bias in the data.

For the main user study, the participant's partner was fixed based on the feedback of the pilot study and he was asked not to express body gestures, face expressions, and speak verbally with the participant during the face-to-face interpersonal experiment. Moreover it is also found that most users in the pilot study preferred a positive sound and perfume stimulus and answered the positive impressions on their communication partners. It caused many difficulties to know how the auditory and olfactory stimuli from the system exactly affected their impression in the analysis of the data. 
Thus the sound and smell stimuli samples were also fixed with negative impressions and not matched impressions with the fixed partner. The author decided to set more contrastive impressions of the fixed sound and perfume stimuli samples to the fixed partner in order to easily track the impressions from the fixed sound and perfume stimuli samples.

For the first step, to know the difference of impression affections between sound and perfume stimuli, the author decided to test it separately. Each group was divided further into three divisions in the main user study. The first was the control and received no stimuli, the second received only set sound stimuli, and the third received only set smell stimuli.

The contrastive sound and perfume stimuli samples were selected from the study of the extracted sensible vocabularies of ten sounds and eight perfume samples. For the negative sound sample, Bouchard's Sound Sample / P03.wav (in the threat section of Vieilard's research) was selected and for the perfume sample, floral perfume / rose sample was selected since all perfume samples had positive images and womanlike fragrance was expected to give more contrastive impressions from the fixed male partner by referencing the study of the extracted sensible vocabularies of ten sounds and eight perfume samples.

The way of the analysis of impression affections that the author used in the pilot study was too subjective. There were biases between the verbal expression of impression on the participant's partner and sensible vocabularies that were extracted from the stimuli samples. To find the objective way to explain the result of the relationship between simuli samples and partners impression, Semantic Differential (SD), which is statistically collecting data by using the self-reporting method, was decided upon to use as the measuring tool of the main user study.

Table 1. The Structure of Huma's Semantic Differential

\begin{tabular}{|c|c|}
\hline Dimensions & Impressions (Left :1 right:7) \\
\hline Sociability & $\begin{array}{l}\text { cold - warm } \\
\text { unfriendly - friendly } \\
\text { unpleasant - pleasant } \\
\text { distant - close } \\
\text { pessimistic - optimistic }\end{array}$ \\
\hline Ethics & $\begin{array}{l}\text { unfair - fair } \\
\text { insincere - sincere } \\
\text { dishonest - honest } \\
\text { incorrect - correct }\end{array}$ \\
\hline Power & $\begin{array}{l}\text { obedient - independent } \\
\text { cowardly - courageous } \\
\text { weak - powerful } \\
\text { shy - bold } \\
\text { undetermined - determined }\end{array}$ \\
\hline Activity & $\begin{array}{l}\text { passive - active } \\
\text { apathetic - energetic } \\
\text { static - dynamic }\end{array}$ \\
\hline
\end{tabular}

Huma [25] used SD research to study the impression comparison between genders and her categorization of measuring impression was adapted and pretested for impression formation assessment. The author made the online SD survey form based on her four dimensions of impressions (Sociability, Ethics, Power, and Activity) to measure a user's impression. The items contained in each dimension are shown in Table 1. The negative impressions have the lower rating score (from 1) and the positive impressions were have the higher rating score (to 7). 


\subsection{Second Stage- Main User Study}

The data from thirty participants were used in the study ( $40 \%$ female, average age is 24.2). From the pilot user study, it was found that the participant's impressions on their partner were affected by their previous relationships. Thus, the author set two groups in the main user stage based on whether the participants have had a previous relationship or if they were meeting their partner for the first time.

Group A had a previous relationship with the set partner, and Group B has no previous relationship with the set partner. Group A were graduate students and researchers in the laboratory and Group B consisted of participants who were invited for this experiment. Each division had five participants. Before the experiment, each participant was only provided an overview of how the sound and perfume spectacle system works and an explanation of the response format separately. The study was conducted at a lounge where people can normally relax for face-to-face conversation.

Participants always sat on the same sofa facing the fixed partner. They were allowed to wear the spectacle in a way that was comfortable to them. They were then given three minutes to assess the partner as shown in Figure 5. Each participant's interaction was recorded using a video camera placed beside the sofas at an angle facing the participants to check the changes of their facial expressions, any verbal feedback provided during the experiment, and gestures made subconsciously.

After the encounter, each participant was asked to rate their impression about the set partner through an online SD survey form immediately.

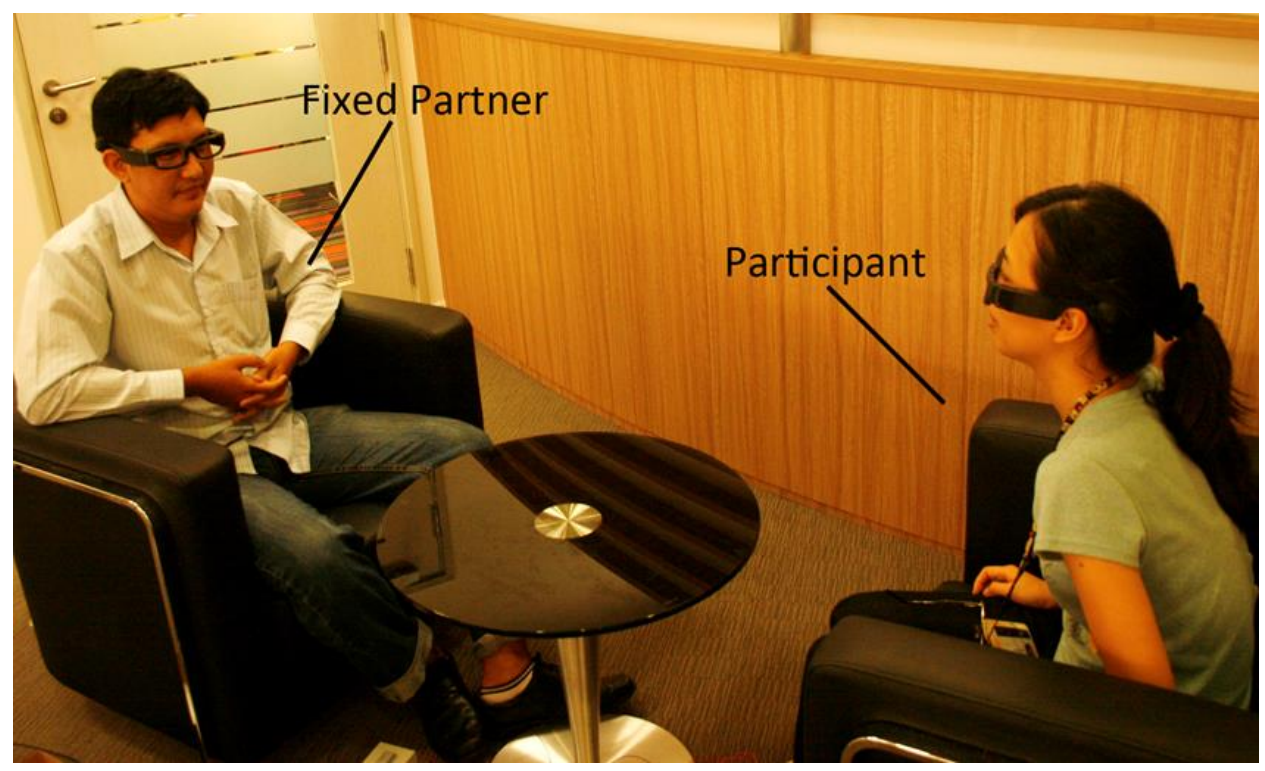

Figure 5. One of Participants is Interacting with the Spectacle During the Second Stage of the User Study 


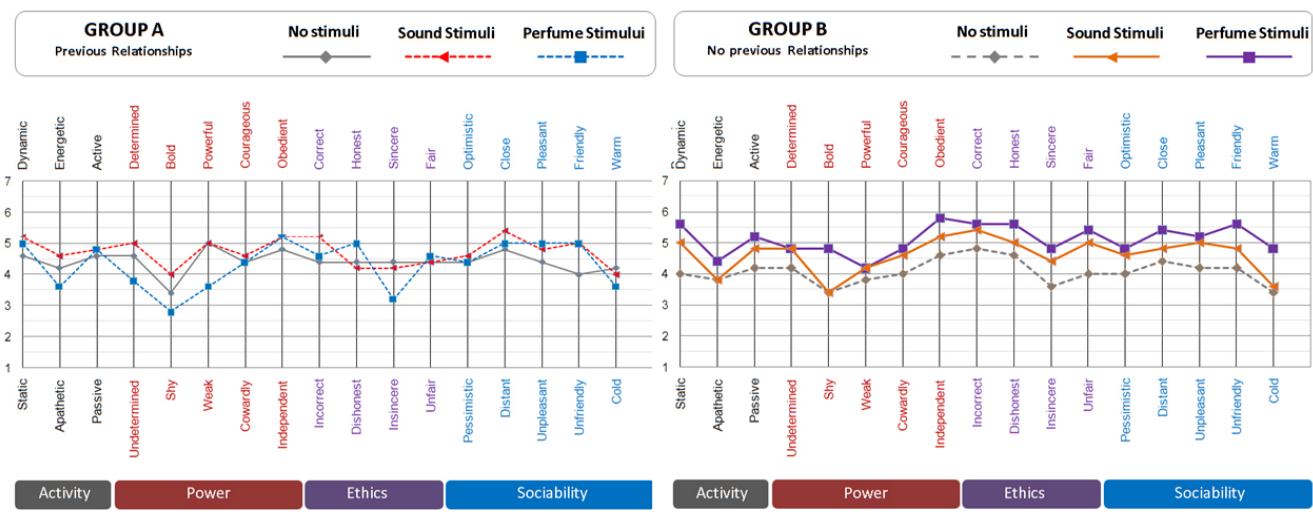

Figure 6. Comparison of Participant's Impressions on the Fixed Partner Based on Group and Stimuli Types

\subsection{Results and Analysis}

The analysis of the results was done based on the comparison graph of mean value as shown in Figure 6 and the mean score of the four impression dimensions across the different groups as shown in Table 2. It was hypothesized that the participants in the division with sound or perfume stimuli would affect to build the impressions of the fixed partner during the face-to-face encounter as compared to the no stimuli. The author wanted to know how subtle sound and perfume stimuli from the spectacle affects people's impressions and which kinds of impressions were affected from the indirect sound and perfume stimulation.

These impressions were measured using a SD with four dimensions Sociability, Ethics, Power and Activity. As Table 2 result shows, the impression on the fixed partner from participants through the fixed sound and perfume stimuli in Group B, who did not have a previous relationship, were rated with the higher scale mean values than those from.

Moreover, as Figure 6 shows, for Group B, the sound and perfume stimuli lines were higher than no stimuli line in all impression dimensions of Group B. It explains the participants in Group B appeared to be positively affected by the partner regardless of the type of stimulus. It was assumed that Group B suffered from the novelty effect; they did not completely understand the subtleties of the negative stimulations but were more fascinated by the new experiences while meeting the new partner.

Table 2. The Mean Scores of the Four Dimensions across the Different Groups (the Bold Fonts Mean the Meaningful Data that Show Over 1.00 Differences from the No Stimuli Data)

\begin{tabular}{|c|c|c|c|c|c|}
\hline Group & Stimuli & Sociability & \multicolumn{1}{c|}{ Ethics } & \multicolumn{1}{c|}{ Power } & Activity \\
\hline \multirow{3}{*}{ A } & None & 4.36 & 4.45 & 4.44 & 4.47 \\
\cline { 2 - 6 } & Sound & 4.76 & 4.75 & 4.76 & 4.87 \\
\cline { 2 - 6 } & Perfume & 4.40 & 4.5 & 3.96 & 4.47 \\
\hline \multirow{3}{*}{ B } & None & 4.12 & 4.35 & 4.00 & 4.00 \\
\cline { 2 - 6 } & Sound & 4.56 & 4.80 & 4.36 & 4.53 \\
\cline { 2 - 6 } & Perfume & 5.16 & 5.05 & 4.84 & 5.07 \\
\hline
\end{tabular}

However, for Group A, who have a previous relationship, the Active, Ethics and Power dimensions were negatively affected by perfume stimuli and all dimensions of impression were very slightly affected by sound stimuli positively. The author assumed that the 
woman like fragrance stimuli with to the fixed male partner might cause some confusion on his previous manly images with new womanlike images to Group A.

As a result, the subtle auditory and olfactory stimuli from the sound and perfume spectacle system can be effectively used in building a positive impression, especially for the first encounter, and it also can potentially affect their social interaction and relationships. And as Group A results show, it can be potentially used to control the wearer's impressions by controlling the stimuli types, if they have previous relation-ships.

\subsection{Limitations and Future Works}

However, the impression during face-to-face interpersonal encounter is formed by many diverse and complex cues and also affected by the feelings which consist of subjective and emotional connections. In short, it is difficult to assert this result can confirm to every scenario and the following limitations were found in the user study:

1) The need to investigate the biases that effect user's impression during face-to-face interpersonal interactions. The user study might also be affected by the user's age, gender and race.

2) There were a low number of participants and this may not justify the use of inferential statistics. Therefore, the interpretation of the results needs to be further empirically tested after more data has been gathered.

3) The author only used two selected sound (S4) and perfume (F1) stimuli samples and the author controlled other bias that can be used in normal face-to-face communication to see the big contrast data in the main user study. It is needed to consider using other sound and perfume stimuli samples and studying the effect of the combination of auditory and olfactory stimulus tests in a normal face-to-face communication environment.

4) Previous relationships formed about someone are very subjective. In the study, the author assumed that the participants, who had met the set partner into a single Group A, can be grouped though this may introduce bias in-to the data.

\section{Conclusions}

Sound and smell can evoke images, emotions, memories, and associations that they can substantially affect us more than even words. In addition, sounds and smells can unconsciously help in creating a mental impression of another person's identity and personality during a face-to-face interpersonal encounter and each unique sound and smell may aid in the memory of one another.

This paper shows the design of the sound and perfume spectacle system, which was designed for building positive impression and expression of unique identities using auditory and olfactory stimulation during face-to-face communication. The user can set his or her own personal sound and smell IDs using mobile phone and when a user begins a face-toface conversation with another person, their sound and perfume preferences are shared between the two systems. The partner's information is used to trigger the sound and perfume actuators in the user's glasses.

To know how the system affects in building a positive impression using subtle sound and smell stimulations, the user studies were conducted and the results show that the sound and perfume spectacle system can help a user affect their impression positively for another person through indirect sound and fragrance stimulation, especially during a first encounter. Potential feasibility to manage the impression by controlling indirect sound and perfume sample stimuli was found in the people who have the previous relationships each other.

To adapt this idea to the real normal daily life, this paper also explores the usage scenarios and natural design forms of electronic sound and smell accessory for the normal lifestyle. The impressive and memorable auditory and olfactory experience from the elec- 
tronic sound and perfume actuation from wearable accessory is working with mobile phone applications and it can be scaled and integrated with many different interaction scenarios with content, objects and spaces. It can enrich consumer electronics through sound and perfume actuation for personal branding.

\section{Acknowledgments}

This work was supported by the Sogang University Research Grant of 2015 (201510001.01)

\section{References}

[1] Y. Choi, R. Parsani, X. Roman, A. V. Pandey, A. D. Cheok, "Sound Perfume: Building Positive Impression during Face-to-Face Communication," SIGGRAPH Asia 2012 Emerging Technologies, Singapore, Singapore, (2012), November 28 - December 01.

[2] Y Amichai-Hamburger, "Loneliness and Internet use," Computers in Human Behavior, vol. 19, no. 1,( 2003). pp. 71-80.

[3] Y. Choi, "Sound Perfume: Designing a Wearable Sound and Fragrance Media for Face-to-Face Interpersonal Interaction," International Conference on Advances in Computer Entertainment Technology, Lisbon, Portugal , (2011) November 08 - 11 .

[4] B. Emsenhuber, "The Olfactory Medium - Smell in Human-Computer Interaction," Science, Technology \& Innovation Studies, vol. 7, no. 1, (2011), pp. 47-64.

[5] B. Emsenhuber, "The Olfactory Medium Science," Technology Innovation Studies, vol. 7, no. 1, (2011) pp. 47-64.

[6] C. Pornpanomchai, A. Threekhunprapa, K. Pongrasamiroj, and P. Sukklay, "SUBSMELL: Multimedia with a Simple Olfactory Display," Advances in Image and Video Technology Tokyo, Japan, (2009), pp. 462-472, January 13-16.

[7] T. Yamada, S. Yokoyama, T. Tanikawa, K. Hirota, and M. Hirose, ”Wearable Olfactory Display: Using Odor in Outdoor Environment," Virtual Reality Conference, Alexandria, Virginia, USA, (2006), March 25-29.

[8] S. Nayak, B. L. Wheeler, S. C. Shiflett, S. Agostinelli, "Effect of music therapy on mood and social interaction among individuals with acute traumatic brain injury and stroke," Rehabilitation Psychology , vol. 45, no. 3, (2000), pp. 274-283.

[9] W.L. Magee, J.W. Davidson, "The effect of music therapy on mood states in neurological patients: A pilot study," Journal of Music Therapy, vol. 39, no. 1, (2002), pp. 20-29.

[10] P. N. Juslin, D. V. Lilestrom, G. Barrades, A. Silva, "An experience sampling study of emotional reactions to music: Listener, music, and situation," Emotion, vol. 9, no. 5, (2008), pp. 668-683.

[11] K. Fox, "The Smell Report An overview of facts and findings", Oxford: Social Issues Research Center, (2007), pp. 24-56..

[12] A. Cann, and D. Ross, "Olfactory stimuli as context cues in human memory," American Journal of Psychology, vol. 102, no. 1,(1989), pp. 91-102.

[13] Y. Yeshurun, H. Lapid, Y. Dudai, and N. Sobel, "The Privileged Brain Representation of First Olfactory Associations," Current Biology, vol. 19, no.21, (2009), pp 1869-1874.

[14] T. Sacco and B. Sacchetti, "Role of Secondary Sensory Cortices in Emotional Memory Storage and Retrieval in Rats," Science, vol.329, no.5992, (2010), pp 649-656.

[15] S. Brewster, D. McGookin, and C. Miller, "Olfoto: designing a smell-based interaction," CHI 2006, , Montréal, Québec, Canada. (2006), April 22-28.

[16] F. Nakaizumi, H. Noma, K. Hosaka, and Y. Yanagida, "SpotScents: A Novel Method of Natural Scent Delivery Using Multiple Scent Projectors," Virtual Reality Conference, Alexandria, Virginia, USA, (2006), March 25-29.

[17] J. Tillotson, "Scentsory Design Scent Whisper and Fashion Fluidics," Communications in Computer and Information Science, vol. 7, (2008), pp 403-417.

[18] T. Narumi, T. Kajinami, T. Tanikawa, and M. Hirose, "Meta cookie," Siggraph 2010, Los Angeles, CA, USA, (2010), July 26 - 30.

[19] D.A. Washburn and L.M. Jones, "Could Olfactory Displays Improve Data Visual-ization?" Computing in Science and Engineering, vol.6, no. 6, (2004), pp 80-83.

[20] R. W. Woodings, D. D. Joos, T. Clifton, and C. D. Knutson, "Rapid Heterogeneous Ad Hoc Connection Establishment: Accelerating Bluetooth Inquiry Using IrDA", Wireless Communications and Networking Conference, Orlando, Florida, USA, (2002), March 17-21.

[21] J. T. Tedeschi, "Impression management theory and social psychological research", Academic Press, New York, (1981).

[22] S. Vieilard, "Happy, sad, scary and peaceful musical excerpts for research on emotions," Journal of Cognition and Emotion, vol. 22, no. 4, (2008), pp 720-752. 
[23] J.E. Parry, "The chemistry of essential oils and artificial perfumes", Greenwood son, London, (1908).

[24] M. Zarzo and D. T. Stanton, "Understanding the underlying dimensions in perfumers odor perception space as a basis for developing meaningful odor maps," Attention, Perception, \& Psychophysics, vol. 71, no. 2, (2009), pp.225-247.

[25] B. Huma, "Gender Differences in Impression Formation," Journal of Comparative Research in Anthropology and Sociology, vol. 1, no. 1, (2010), pp. 57-72.

\section{Author}

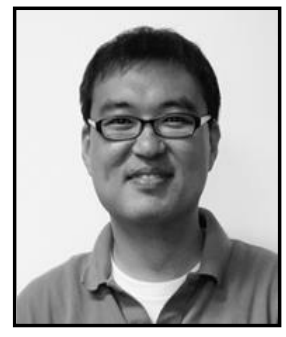

Yongsoon Choi, is an assistant professor at the Art \& Technology Major at Sogang University, Korea. His researches focus on multimodal and cross-modal interactions for wearable media \& food media and affective wearable interfaces $\&$ interactions. 
International Journal of Multimedia and Ubiquitous Engineering

Vol.12, No.4 (2017) 